Cinémas

Revue d'études cinématographiques

Journal of Film Studies

\title{
Document
}

\section{Congrès de 1955. Groupe VI}

Volume 19, numéro 2-3, printemps 2009

La filmologie, de nouveau

URI : https://id.erudit.org/iderudit/037559ar

DOI : https://doi.org/10.7202/037559ar

Aller au sommaire du numéro

Éditeur(s)

Cinémas

ISSN

1181-6945 (imprimé)

1705-6500 (numérique)

Découvrir la revue

Citer ce document

(2009). Document / Congrès de 1955. Groupe VI. Cinémas, 19(2-3), 327-331.

https://doi.org/10.7202/037559ar d'utilisation que vous pouvez consulter en ligne.

https://apropos.erudit.org/fr/usagers/politique-dutilisation/ 


\section{Congrès de 1955 \\ Groupe VI}

Président P. Francastel

Dimanche 20 février (matin)

Présents: Pierre Francastel, Prof. Tanpinar (Istanbul), M. Jeannesson (Paris), M. Fuzellier (Idhec), Dr Agnès Bleier Brody (Vienne), M. Léglise (CNC), Prof. Van Boerge (Université de Vienne), M. J. Duvignaud (attaché de recherche CNRS), M. Soriano (Paris)

M. Francastel ouvre la séance en indiquant qu'il vient de recevoir personnellement une communication de M. Bayer sur «l'intelligibilité du temps filmique». Il en donne lecture. La communication traite de l'intelligibilité du temps filmique par les divers types psychologiques ou sociaux des spectateurs et suggère une étude de la manière dont est comprise, dans certains films et par certains spectateurs, la dislocation du temps et la causalité présentes dans le film.

M. Francastel signale également quelques communications pouvant intéresser les membres du Groupe VI et servir de point de contact avec les autres groupes: celles de MM. Galifret et Hansell, celles de M. et $\mathrm{M}^{\text {me }}$ Zazzo.

M. Fuzellier fait remarquer l'extrême variété des sujets traités.

M. Francastel: Il faut essayer de dégager deux ou trois problèmes en vue de présenter un certain nombre d'observations sur les possibilités de travail. Demande de suggérer certains centres d'intérêt, et rappelle que le sujet proposé était l'étude des rapports entre le cinéma et les autres arts. Il pense qu'il faut suivre dans une certaine mesure l'ordre du jour.

M. Fuzellier rappelle les thèmes généraux et pense qu'une voie d'approche serait de partir de l'expression filmique en comparaison avec les ouvres qui sont ou non inscrites dans le temps ${ }^{1}$.

$\mathrm{M}^{\mathrm{me}}$ Bleier Brody ${ }^{2}$ voudrait voir discutées les méthodes scientifiques pouvant être appliquées à l'étude de ces problèmes 
spéciaux au groupe et les autres recherches, ainsi qu'avec le travail pratique. Elle souhaite une comparaison entre l'histoire des arts dramatique et filmique depuis les âges de la présentation à caractère religieux. Il faudrait suggérer au Congrès que la méthode de recherche filmique soit poursuivie à partir de l'étude du film au milieu d'autres formes de spectacles. Il faudrait, ditelle, suggérer aux organismes de recherche filmologique d'aller dans ce sens, et de se demander également en quoi les recherches du groupe sur la spécificité de l'expression filmique peuvent servir les techniciens des autres arts. Il serait également intéressant de savoir quelle est l'influence sur le public de l'art spécial du cinéma, où se produit, avec l'acteur qui se présente presque de la même façon que les silhouettes, une séparation entre le personnage et sa voix. Le public dissocie l'unité humaine du personnage en ces deux éléments. Il y a une sorte de réaction schizophrène de la part du public, semblable à ce qui était le cas pour le théâtre de marionnettes. Cet aspect permettrait aux techniciens de tenir compte des réactions du public.

M. Francastel verrait un rapprochement entre ces idées et les travaux du Groupe III qui se préoccupe des réactions du public. Il n'est pas d'accord avec M. Fuzellier : tout art est spectacle et le temps intervient partout, même en peinture.

$\mathbf{M}^{\text {me }}$ Bleier Brody cite le Prof. Gregor, pour lequel l'idée temps-espace est un problème central. D'après lui, la musique est un art temps-espace, pour le cinéma c'est l'inverse.

M. Léglise : Le cinéma rassemble les autres arts.

M. Francastel insiste sur le fait que la peinture n'est pas un art immobile, la position de [Canudo] n'est plus soutenable, elle est dépassée. En réponse à $M$. Léglise qui cite un extrait de $L^{\prime E ́ n e ́ i d e ~}{ }^{3}$, il explique que c'est là un problème de modes de présentation.

M. Boerge établit une comparaison théâtre-film avec HuisClos [de Jean-Paul Sartre] où le présent est dramatique. Il estime que de ce point de vue le film [de Jacqueline Audry] ${ }^{4}$ est plus intéressant que la pièce.

M. Duvignaud n'est pas d'accord car la transposition ici a fait disparaître des éléments dramatiques et a causé un rétrécissement. 


\section{Document}

M. Boerge: Mais Huis clos est un exemple pour le problème central qu'est le temps.

$\mathbf{M}^{\mathrm{me}}$ Bleier Brody demande si pour tous les arts le temps est le même ou non.

M. Jeannesson: Si on emploie la méthode de l'esthétique comparée, on débouchera sur les caractères spécifiques du cinéma, mais on peut aussi les perdre.

M. Francastel : En somme, il s'agit de savoir s'il y a des notions fixes d'espace et de temps dont les différents arts ne sont qu'une illustration, ou s'il y a création d'un temps dans chaque forme dramatique.

M. Boerge: Il faut savoir ce qu'est le film, il souligne la spécificité absolue de l'expression filmique.

$M^{\text {me }}$ Bleier Brody n'est pas d'accord, théâtre et cinéma ont en commun le caractère de divertissement, la spécificité du film n'est pas le film tout entier. Il y a transposition d'un art à l'autre.

M. Léglise: Il convient de rechercher quels sont le domaine et les limites de la transpositon filmique.

M. Francastel cite un ouvrage italien [Ragghianti dans Cinema et arte figurativa] ${ }^{5}$ dont l'auteur dit que le cinéma n'a pas été créé par la caméra à elle seule qui ne suffit pas à expliquer les caractères spécifiques du film, la caméra n’a fait que fournir à la vision filmique les moyens de se réaliser. Il existe une attitude filmique antérieure au cinéma.

M. Duvignaud estime qu'il faut partir de l'analyse concrète de la temporalité créée par le metteur en scène.

M. Francastel répond qu'il n'y a pas une seule solution au problème du temps, mais il faut que le groupe signale l'existence du problème.

M. Soriano: Huis clos dont il a été parlé est un mauvais exemple car chez Sartre temps et espace sont un leitmotiv.

M. Boerge: Le problème commence par les expressionnistes. Il existe une ligne idéale de la spécificité filmique.

M. Fuzellier pense qu'il pourrait y avoir dans le film un temps tragique et un temps comique par exemple. Ainsi Le jour se lève [Marcel Carné, 1938] commence par la fin, et ce procédé de réversibilité du temps a une vocation tragique, la fin connue du spectateur pèse sur tout le film. 


\section{Document}

M. Francastel: Il n'y a pas une forme du film, le film apporte des possibilités d'expression. Une ambiguïté persiste dans la notion de temps qui vient de faire l'objet de la discussion, car les appréciations ont été techniques aussi bien qu’esthétiques.

Il propose d'envisager maintenant un programme de recherches précises:

1) le problème du pré-cinéma et de l'attitude filmique

2) études concrètes des temps filmiques et des moyens de réalisation.

M. Fuzellier: Comme il y a un pré-cinéma, y a-t-il un temps pré-filmique?

M. Boerge voudrait voir étudiées les qualités immédiates de l'expression cinématographique.

M. Francastel: Il faut ajouter à ce programme la notion de spectacle, et étudier les différentes formes des valeurs pré-filmiques dans les différents spectacles. La spécificité du temps film n’est qu'un aspect du problème, parmi ceux qui peuvent servir de base aux analyses. Il faudrait également évoquer le problème de la succession, et faire des analyses comparatives entre le film et d'autres formes d'art. Il y a donc le problème du temps, le problème de la continuité ou de la succession, enfin le problème de la lecture du film et des autres œuvres d'art. Le film apporte un temps absolument inédit quil faudrait aborder par le découpage. Le découpage filmique est spécifique. Il suggère le terme d'ordonnancement du filmique, ordonnancement qui ne s'est constitué qu'avec le temps ${ }^{6}$.

\section{Note de la rédaction}

Le texte qui précède est la retranscription par François Albera d'un document déposé en archives (dépôt Antoine Borel, Cinémathèque suisse), quil a également annoté et complété. Le texte source est luimême la transcription d'interventions orales non révisées par les auteurs. Dans un certain nombre de cas (en particulier en présence de noms propres mal orthographiés ou mal identifiés), les erreurs manifestes dues à la sténographie ont été corrigées. Toutes les autres interventions apparaissent entre crochets. 


\section{NOTES}

1. Étienne Fuzellier, enseignant en lettres et professeur à l'Idhec, a publié par la suite Cinéma et littérature (Paris, Institut des hautes études cinématographiques, collection "Cours et Publications de l'Idhec», 1957, 2 volumes) repris en 1964 aux Éditions du Cerf (collection " $7^{\mathrm{c}}$ art $\left.»\right)$.

2. Agnès Bleier Brody, critique autrichienne qui a également travaillé pour la cinémathèque de Vienne.

3. Paul Léglise est l'auteur de Une xuvre de pré-cinéma. L'Énéide. Essai d'analyse filmique du premier Chant, Paris, Nouvelles Éditions Debresse, 1958 (Préface de B. Georgin).

4. Huis clos (France, 1954), réal.: Jacqueline Audry, scén. et dial.: Pierre Laroche d'après Jean-Paul Sartre, musique: Joseph Kosma, interprétation: Arletty, Gaby Sylvia, Frank Villard, Nicole Courcel, Danièle Delorme, Yves Deniaud, Jean Debucourt, Louis de Funès, Paul Frankeur, Giani Esposito.

5. Carlo L. Ragghianti, Cinema arte figurativa, Torino, Einaudi, 1952. Dans le cours du 9 avril 1956, Francastel reprend cette référence.

6. Dimanche après-midi est abordé le problème de l'adaptation (avec, outre les personnes indiquées ci-dessus, la participation de Clara Malraux). Dans une lettre à Francastel Roland Barthes s'excuse de ne pouvoir participer au travail du Groupe VI en raison d'une bronchite et de fièvre. "Ma première sortie sera pour le Congrès", affirme-t-il dans sa lettre. 